\title{
CLASS, DIMENSION AND LENGTH IN NILPOTENT LIE ALGEBRAS*
}

\author{
LISA WOOD BRADLEY ${ }^{\dagger}$ AND ERNIE L. STITZINGER ${ }^{\ddagger}$
}

\begin{abstract}
The problem of finding the smallest order of a $p$-group of a given derived length has a long history. Nilpotent Lie algebra versions of this and related problems are considered. Thus, the smallest order of a $p$-group is replaced by the smallest dimension of a nilpotent Lie algebra. For each length $t$, an upper bound for this smallest dimension is found. Also, it is shown that for each $t \geq 5$ there is a two generated Lie algebra of nilpotent class $d=21\left(2^{t-5}\right)$ whose derived length is $t$. For two generated Lie algebras, this result is best possible. Results for small $t$ are also found. The results are obtained by constructing Lie algebras of strictly upper triangular matrices.
\end{abstract}

Key words. Two generated, Three generated.

AMS subject classifications. 17B30.

1. Introduction. Glasby in [4] considered the solvable length of subgroups of unipotent matrices $U=U(n)$ of size $n$. In particular, given an integer $t$, what is the smallest matrix size, $n$, which supports a subgroup of $U$ of length $t$. Clearly for $U(n)$ itself, $n=2^{t-1}+1$. Glasby then studies the problem when one restricts the number, $r$, of generators. When $r=3$ or more, the same bound is found and it is best possible. The $r=2$ case is also solved. There are similar kinds of problems which have long been investigated. A problem dating back to Burnside $[1,2]$ is to find the order of the smallest $p$-group which has length $t$. Substantial contributions to this and many more problems are found in [5]. Recent results and an excellent summary can be found in [3]. One also can seek the smallest nilpotent class for $p$-groups of fixed length $t$. Generally, the answer is $2^{t-1}$ with $U(n)$ providing an example. As above, this question can be posed with restrictions on the number of generators. In this work we discuss Lie algebra analogues to these problems. In some cases, stronger results no doubt exist.

2. Length in Two Generated Lie Algebras. Let $L=F[x, y]$ be the free Lie algebra on two generators $x$ and $y$. Let $L, L^{(1)}, \ldots, L^{(n)}$ be the derived series and $L, L^{2}, \ldots, L^{n}$ be the lower central series for $L$. Recall that $L$ has class $n$ if the product of $n+1$ elements is always 0 but there is a product of $n$ elements which is not $0 . L$ has length $t$ if $t$ is the smallest integer such that $L^{(t)}=0$. By a word in $L$ of width $n$ we mean a monic monomial in $x$ and $y$ with $n$ factors. $L^{n}$ is all linear combinations of words of width $n$ and greater. For each length $t$, let $n_{t}$ be the width of the shortest word in $L^{(t-1)}$. Then the words in $L^{(t)}$ have width greater than or equal to $2 n_{t}$. Since $2 n_{t} \geq n_{t}+1, L^{(t)}$ is contained in $L^{n_{t}+1}$ Then $L / L^{n_{t}+1}$ has class $n_{t}$ and length $t$. When $t=2, n_{2}=2$ and the word is $w_{2}=[x, y]$. Therefore $L / L^{3}$ has length 2 and class 2. Both $w_{3}=[[x, y], x]$ and $w_{3}^{\prime}=[[x, y], y]$ are also in $L^{(1)}$. Then $w_{5}=\left[w_{2}, w_{3}\right]$

* Received by the editors 29 May 2007. Accepted for publication 26 November 2007. Handling Editor: Robert Guralnick.

†IBM, 4205 S. Miami Blvd. 502/J227 Durham, NC 27703, USA (lbradley@us.ibm.com).

¥Mathematics Department, NC State University, Raleigh, NC 27695, USA (stitz@ncsu.edu). 
and $w_{5}^{\prime}=\left[w_{2}, w_{3}^{\prime}\right]$ are in $L^{(2)}$. Hence $n_{3}=5, L / L^{6}$ has class 5 and length 3 and $w_{6}=\left[w_{3}, w_{3}^{\prime}\right] \in L^{(2)}$. Then $n_{4}=10$ with $w_{10}=\left[w_{5}, w_{5}^{\prime}\right] \in L^{(3)}$ and $w_{10}$ is the only monomial of width 10 in $L^{(3)}$. Also $w_{11}=\left[w_{5}, w_{6}\right]$ and $w_{11}^{\prime}=\left[w_{5}, w_{6}^{\prime}\right]$ are in $L^{(3)}$. Then $w_{21}=\left[w_{10}, w_{11}\right], w_{21}^{\prime}=\left[w_{10}, w_{11}^{\prime}\right]$ and $w_{22}=\left[w_{11}, w_{11}^{\prime}\right] \in L^{(4)}$, hence $n_{5}=21$. We claim that $n_{t} \geq 21\left(2^{t-5}\right)$ when $t \geq 5$ Assume the result for $t$. The smallest width of a word in $L^{(t+1)}$ is at least $2\left(2^{t-5}\right)$. Therefore $n_{t+1} \geq 2^{t+1-5}$. It remains to show that these inequalities are equalities.

The results shown thus far are collected as

Lemma 2.1. Let $L=F[x, y]$ be the free Lie algebra on two generators $x$ and $y$. Then for $t$, the lower bound $m$ such that $L / L^{m}$ has length $t$ is given in the following table. $L / L^{m}$ has class $n_{t}=m-1$ which is a lower bound for the class. Hence for $L / L^{m}$ to have length $t, m \geq 21\left(2^{(t-5)}\right)+1$, when $t \geq 5$.

\begin{tabular}{|c|c|c|}
\hline length $=t$ & class $=n_{t}$ & $m$ \\
\hline 2 & 2 & 3 \\
\hline 3 & 5 & 6 \\
\hline 4 & 10 & 11 \\
\hline$t \geq 5$ & $21\left(2^{t-5}\right)$ & $21\left(2^{t-5}\right)+1$ \\
\hline
\end{tabular}

We will show that the values in the second column in the table are met and, therefore, the smallest possible class for any 2-generated Lie algebra of length $t$ appears in the second column of the above table.

Let $A$ and $B$ be matrices in $T=T(n)$, the strictly upper triangular $n$ by $n$ matrices. If $w(x, y)$ is a word of width $k$ in $\mathrm{F}[x, y]$, then $w(A, B)$ is computed in the associative envelope, $T^{*}$, of $T$. Suppose that both $A$ and $B$ are the sum of elementary matrices, $E_{i, i+1}$, where each $E_{i, i+1}$ occurs in $A$ or $B$ but not both. Each monomial in the expansion of $w(A, B)$ is either 0 or the product of consecutive elementary matrices in which case the product is $E_{i, i+k}$. Now suppose that $k=n$. Then $w(A, B)=0$. If $k=n-1$, then $w(A, B) \neq 0$ implies that the term $E_{1,2} E_{2,3} \ldots E_{n-1, n}=E_{1, n}$ appears. Such a product only exists if the number of times $x$ appears in $w(x, y)$ coincides with the number of the $E_{i, i+1}$ which appear in $A$ and likewise for $y$ and $B$.

In the following we enlarge matrices by the process now described. Suppose that $A$ and $A_{1}$ are as in the last paragraph. Concatenate $A$ and $A_{1}$ by enlarging $A$ to a $2 n-1$ by $2 n-1$ matrix by adding $n-1$ rows and columns of 0 's to the bottom and right borders and enlarging $A_{1}$ by adding $n-1$ columns of 0 's to the top and left borders. Add these new matrices to get $A^{\prime}$. Thus an $n$ by $n E_{i, i+1}$ occurs in $A$ if and only if a $2 n-1$ by $2 n-1 E_{i, i+1}$ occurs in $A^{\prime}$ and an $n$ by $n E_{i, i+1}$ occurs in $A_{1}$ if and only if a $2 n-1$ by $2 n-1 E_{i+n-1, i+n}$ occurs in $A^{\prime}$. Construct $B^{\prime}$ from $B$ and $B_{1}$ in the same way. The $(1, n)$ position in $w(A, B)$ agrees with the $(1, n)$ position in $w\left(A^{\prime}, B^{\prime}\right)$ for they both depend solely on the appearance of $E_{1,2} E_{2,3} \ldots E_{n-1, n}=E_{1, n}$ in the expansion of $w(A, B)$.

LEMma 2.2. $E_{1, n}$ has the same coefficient in the expansion of $w(A, B)$ as does (the $2 n-1$ by $2 n-1$ matrix) $E_{1, n}$ in $w\left(A^{\prime}, B^{\prime}\right)$. This coefficient is 0 unless the number of times $x$ occurs in $w(x, y)$ is the same as the number of $E_{i, i+1}$ in $A$ and likewise for $y$ and $B$. 
Similarly,

LEMma 2.3. $E_{1, n}$ has the same coefficient in the expansion of $w\left(A_{1}, B_{1}\right)$ as does (the $2 n-1$ by $2 n-1$ matrix) $E_{n, 2 n-1}$ in $w\left(A^{\prime}, B^{\prime}\right)$. This coefficient is 0 unless the number of times $x$ occurs in $w(x, y)$ is the same as the number of $E_{i, i+1}$ in $A_{1}$ and likewise for $y$ and $B_{1}$.

LEMMA 2.4. If $w(x, y)$ and $w_{1}(x, y)$ have the same width and a different number of $x$ 's, then either the $(1, n)$ and $(n, 2 n-1)$ positions for $w\left(A^{\prime}, B^{\prime}\right)$ or the $(1, n)$ and $(n, 2 n-1)$ positions $w_{1}\left(A^{\prime}, B^{\prime}\right)$ are 0 .

Let $T$ be the Lie algebra generated by $A$ and $B$, two strictly upper triangular matrices of size $n$. Then $T$ is the homomorphic image of $L=F[x, y]$ where $w(x, y)$ goes to $w(A, B)$. Hence the inequalities in 2.1 hold in $T$ as well. We construct examples of $T$ that show that these are equalities. Thus they are equalities in $L$ and we have equalities for the smallest class for a 2-generated Lie algebra that will support a given solvable length. These are given in 2.1 with the replacement of the inequalities by equalities.

THEOREM 2.5. For each $t$, there is a 2-generated Lie algebra of nilpotent class $d$ whose derived length is $t$ as in the following table. An example can be found in $T(d+1)$ and this is the smallest size possible. Also, $d$ is the smallest possible.

\begin{tabular}{|c|c|}
\hline length $=t$ & class $=d$ \\
\hline 2 & 2 \\
\hline 3 & 5 \\
\hline 4 & 10 \\
\hline$t \geq 5$ & $21\left(2^{t-5}\right)$ \\
\hline
\end{tabular}

Proof. For $t=3$. In $T(6)$, let $A=E_{1,2}+E_{2,3}+E_{4,5}$ and $B=E_{3,4}+E_{5.6}$. Let $w_{5}((x, y))=[[[y, x], x],[y, x]]$. Then $w_{5}(A, B)=E_{1,6}$. Therefore for $t=3, d=5$ and the smallest matrix size is $n=6$.

For $t=4$. In $T(11)$, let $A=E_{3,4}+E_{4,5}+E_{6,7}+E_{7,8}+E_{10,11}$ and $B=$ $E_{1,2}+E_{2,3}+E_{5,6}+E_{8,9}+E_{9,10}$. Let $w_{10}(x, y)=[[[[y, x], y],[y, x]],[[[y, x], x],[y, x]]]$ Then $w_{10}(A, B)=E_{1,11}$. Therefore, $t=4, d=10$ and $n=11$.

For $t=5$. In $T(22)$, let $A_{1}=E_{4,5}+E_{5,6}+E_{7,8}+E_{8,9}+E_{11,12}+E_{14,15}+E_{15,16}+$ $E_{17,18}+E_{18,19}+E_{21,22}$ and $B_{1}=E_{1,2}+E_{2,3}+E_{3,4}+E_{6,7}+E_{9,10}+E_{10,11}+E_{12,13}+$ $E_{13,14}+E_{16,17}+E_{19,20}+E_{20,21}$. Set $t_{1}=[[[[y, x] y] y],[y, x]], t_{2}=[[[y, x], x],[y, x]]$, $t_{3}=[[[y, x], x],[y, x]]$ and $w_{21}^{1}=\left[\left[t_{1}, t_{2}\right],\left[t_{3}, t_{2}\right]\right]$. Then $\left.w_{21}^{1} A_{1}, B_{1}\right)=E_{1,22}$. Hence $t=5, d=21$ and $n=22$.

For further use, two more examples are needed at this level. Let $A_{2}=E_{1,2}+$ $E_{2,3}+E_{6,7}+E_{7,8}+E_{8,9}+E_{11,12}+E_{14,15}+E_{15,16}+E_{17,18}+E_{18,19}+E_{21,22}$ and $B_{2}=E_{3,4}+E_{4,5}+E_{5,6}+E_{9,10}+E_{10,11}+E_{12,13}+E_{13,14}+E_{16,17}+E_{19,20}+E_{20,21}$. Set $t_{4}=[[[[y, x], x], y],[y, x]]$ and $w_{21}^{2}=\left[\left[t_{4}, t_{2}\right],\left[t_{3}, t_{2}\right]\right]$. Then $w_{21}^{2}\left(A_{2}, B_{2}\right)=E_{1,22}$. Finally, let $A_{3}=E_{1,2}+E_{2,3}+E_{3,4}+E_{6,7}+E_{7,8}+E_{8,9}+E_{11,12}+E_{14,15}+E_{15,16}+$ $E_{17,18}+E_{18,19}+E_{21,22}$ and $B_{3}=E_{4,5}+E_{5,6}+E_{9,10}+E_{10,11}+E_{12,13}+E_{13,14}+$ $E_{16,17}+E_{19,20}+E_{20,21}$. Set $t_{5}=[[[[y, x], x], x],[y, x]]$ and $w_{21}^{3}=\left[\left[t_{5}, t_{2}\right],\left[t_{3}, t_{2}\right]\right]$. Then $w_{21}^{3}\left(A_{3}, B_{3}\right)=E_{1,22}$. Note that the exponent of each $w$ is congruent mod 3 to the number of $x$ 's in that $w$. 
For $t=6$. We use the $A$ 's, $B$ 's and $w$ 's from the $t=5$ case. As explained above, concatenate $A_{1}$ and $A_{2}$ forming $A_{3}^{\prime}$ and $B_{1}$ and $B_{2}$ forming $B_{3}^{\prime}$. Let $w_{42}^{3}(x, y)=$ $\left[w_{21}^{1}(x, y), w_{21}^{2}(x, y)\right]$. Then

$$
w_{42}^{3}\left(A_{3}^{\prime}, B_{3}^{\prime}\right)=w_{21}^{1}\left(A_{3}^{\prime}, B_{3}^{\prime}\right) w_{21}^{2}\left(A_{3}^{\prime}, B_{3}^{\prime}\right)-w_{21}^{2}\left(A_{3}^{\prime}, B_{3}^{\prime}\right) w_{21}^{1}\left(A_{3}^{\prime}, B_{3}^{\prime}\right) .
$$

Since $A_{3}^{\prime}$ and $B_{3}^{\prime}$ are strictly upper triangular matrices entering in a product with 42 terms, the only possible non-zero position is $(1,43)$. From the $t=5$ case, $w_{21}^{1}\left(A_{3}^{\prime}, B_{3}^{\prime}\right)$ has a 1 in the $(1,43)$ position and $w_{21}^{2}\left(A_{3}^{\prime}, B_{3}^{\prime}\right)=0$ since $w_{21}^{2}$ and $w_{21}^{1}$ have a different number of $x$ 's. In the $(22,43)$ position, $w_{21}^{2}\left(A_{3}^{\prime}, B_{3}^{\prime}\right)$ is 1 from the $t=5$ case while $w_{21}^{1}\left(A_{3}^{\prime}, B_{3}^{\prime}\right)=0$. Therefore $w_{42}^{3}\left(A_{3}^{\prime}, B_{3}^{\prime}\right)=E_{1,43}$.

The number of $x$ 's in $w_{42}^{3}(x, y)$ is equal to the sum of the number of $x$ 's in $w_{21}^{1}(x, y)$ and the number of $x$ 's in $w_{21}^{2}(x, y)$. Therefore each of these numbers is congruent mod 3 to the exponent of the $w$ from which it comes. In fact, this is the reason for the choice of exponent in the last term.

There are two other cases giving rise to $w_{42}^{1}(x, y)=\left[w_{21}^{1}(x, y), w_{21}^{3}(x, y)\right]$ with $A_{1}^{\prime}$ the concatenation of $A_{1}$ and $A_{3}$ and $B_{1}^{\prime}$ the concatenation of $B_{1}$ and $B_{3}$ and $w_{42}^{2}(x, y)=\left[w_{21}^{2}(x, y), w_{21}^{3}(x, y)\right]$ with $A_{2}^{\prime}$ the concatenation of $A_{2}$ and $A_{3}$ and $B_{2}^{\prime}$ the concatenation of $B_{2}$ and $B_{3}$ with corresponding results as in the last paragraph. In particular, the $(1,43)$ position in $w_{42}^{1}\left(A_{1}^{\prime}, B_{1}^{\prime}\right)$ is 1 or -1 as is the $(1,43)$ position in $w_{42}^{2}\left(A_{2}^{\prime}, B_{2}^{\prime}\right)$. Hence for $t=6, d=42$ and $n=43$. The process is set up to continue with $d=21\left(2^{t-5}\right)$ and $n=21\left(2^{t-5}\right)+1$. $\square$

3. On The Minimal Dimension For a Given Length. Let $d_{j}$ be the $j^{\text {th }}$ super diagonal of $T=T(n)$. Then $T=d_{1}+d_{2}+\ldots+d_{n-1}$ and $T^{(k)}=d_{2^{k}}+\ldots d_{n-1}$. Now $T^{(k-1)} \neq 0$ if and only if $2^{k-1} \leq n-1$. Hence the least $n$ such that $T$ has length $t$ is $n=2^{t-1}+1$ and $\operatorname{dim} T=\frac{(n+1) n}{2}$. We will reduce this dimension by taking a three generated subalgeba of $T$.

Let $F_{i, j}=\sum_{k=0}^{j+3 k \leq n} E_{i+3 k, j+3 k}$ where $i=1,2,3, \ldots$ and $j>i$. Let $L$ be the Lie algebra generated by $F_{1,2}, F_{2,3}$ and $F_{3,4}$. Clearly $F_{i, i+p} F_{j, j+q}=F_{i, p+q+i}$ if $p+i$ is congruent to $j \bmod 3$ and is 0 otherwise. For notational convenience set $A=$ $F_{i, i+p} F_{j, j+q}$ and $B=F_{j, j+q} F_{i, i+p}$ and $s=p+q$.

LEMMA 3.1. If $A$ and $B$ are not both 0 , then $s$ is congruent to 0 mod 3 and $A-B \in d_{s}$.

Proof. Since 3 divides $p+i-j$ and $q+j-i$, it also divides $s=p+q$. Furthermore $A-B=F_{i, i+s}-F_{j, j+s} \in d_{s}$.

Lemma 3.2. If $s$ is congruent to 0 mod 3, then either both $A$ and $B$ are 0 or both are not 0 .

Proof. Assume that $A \neq 0$. Then $s$ divides $p+i-j=s-(q+j-i)$ and $q+j-i$. Hence $B \neq 0$.

Lemma 3.3. $F_{j, 2^{s}+j} \in L^{(s)}$ for $j=1,2,3, \ldots$ while $2^{s}+j \leq n$.

Proof. Note that $F_{j, 2^{s}+j} \in d_{2^{s}}$. When $s=1,\left[F_{1,2}, F_{2,3}\right]=F_{1,3},\left[F_{1,2}, F_{3,4}\right]=$ $-F_{3,5}$ and $\left[F_{2,3}, F_{3,4}\right]=F_{2,4}$ are all in $L^{(1)}$. Assume that $F_{j, j+2^{k}} \in L^{(k)}$ for $j=$ $1,2,3, \ldots$ For $1 \leq i<j \leq 3,\left[F_{i, 2^{k}+i}, F_{j, 2^{k}+j}\right]=F_{i, 2^{k}+i} F_{j, 2^{k}+j}-F_{j, 2^{k}+j} F_{i, 2^{k}+i} \in$ $L^{(k+1)}$. Both terms on the right hand side of this expression will be non-zero exactly when $i$ is congruent to $j+2^{k} \bmod 3$ and $j$ is congruent to $i+2^{k+1} \bmod 3$. If these 
congruences both hold, then three divides $2^{k+1}$ which is a contradiction. Now $i-j$ is congruent to 1 or $2 \bmod 3$; that is, either $i-j$ or $j-i$ is congruent to $2^{k} \bmod$ 3. Thus the above commutator equals one of the terms on the right hand side. By considering the possibilities for $i$ and $j$, the lemma holds.

LEMMA 3.4. $L=<F_{1,2}, F_{2,3}, F_{3,4}>$ in $T(n)$ has length $t$ if $2^{t-1}+1 \leq n<2^{t}+1$. Therefore $n=2^{t-1}+1$ is the smallest $n$ such that $L$ has length $t$ for any 3-generated subalgebra of $T(n)$.

Proof. The smallest possible $n$ is $n=2^{t-1}+1$ for any subalgebra of $T(n)$. If $n \geq 2^{t}+1$, then $L^{(t-1)} \neq 0$ since $F_{1,2^{t-1}+1} \in L^{(t-1)}$. $\square$

We now find $\operatorname{dim} L$. $L=\sum_{j=1}^{n-1} d_{j}^{\prime}$ where $d_{j}^{\prime}=d_{j} \cap L$.

LEMMA 3.5. The following hold

1. $\operatorname{dim} d_{s}^{\prime}=3$ if $s$ is not congruent to $0 \bmod 3, s \neq n-2, n-1$;

2. $\operatorname{dim} d_{s}^{\prime}=2$ if $s$ is congruent to $0 \bmod 3, s \neq n-1$;

3. $\operatorname{dim} d_{n-2}^{\prime}=2$;

4. $\operatorname{dim} d_{n-1}^{\prime}=1$.

Proof. Suppose that $s$ is not congruent to $0 \bmod 3 . d_{s}^{\prime}$ is obtained from terms $\left[F_{i, p+i}, F_{j, q+j}\right]$ where $s=p+q$. Since 3 does not divide $s$, this commutator equals either $F_{i, s+i}$ or $-F_{j, q+j}$, depending on $1 \leq i<j \leq 3$. Hence

$$
d_{s}^{\prime}=<F_{1, s+1}, F_{2, s+2}, F_{3, s+3}>\text {. }
$$

Suppose that $s$ is congruent to $0 \bmod 3$. As in the last paragraph, $d_{s}^{\prime}$ consists of commutators but of the form $F_{i, s+i}-F_{j, s+j}$. In particular we obtain $F_{1, s+1}-F_{2, s+2}$, $F_{2, s+2}-F_{3, s+3}$ and $F_{1, s+1}-F_{3, s+3}$. Since the last term is the sum of the first two, $d_{s}^{\prime}$ has dimension 2. The final two cases are clear.

Lemma 3.6. The dimension of $L$ is

1. $8(k-1)+3$ when $n=3 k$;

2. $8(k-1)+6$ when $n=3 k+1$;

3. $8(k-1)+9$ when $n=3 k+2$.

Proof. Dim $L=3+3+2+3+3+2+\ldots+2+1$ where there are $n-1$ terms in this expression. In all cases, the first $3(k-1)$ terms can be grouped in threes, each having a sum of 8 . If $n=3 k$, there remain $2+1$ in the sum, giving a total of $8(k-1)+3$. If $n=3 k+1$, there remain $3+2+1$ in the sum, giving a total of $8(k-1)+6$. Finally, if $n=3 k+2$, there remain $3+3+2+1$ in the sum, giving a total of $8(k-1)+9$.

THEOREM 3.7. There is a three generated Lie algebra $L$ of strictly upper triangular matrices of size $n=2^{t-1}+1$ and length $t$ and dimension

1. $\left(2^{t+2}-7\right) / 3$ when $t$ is even;

2. $\left(2^{t+2}-5\right) / 3$ when $t$ is odd.

Proof. We have seen that for $L$ to have length $t$, the minimum matrix size is $n=2^{t-1}+1$. If $t$ is even, then $n$ is congruent to $0 \bmod 3$ and if $t$ is odd, then $n$ is congruent to $2 \bmod 3$. Therefore, if $t$ is even then $n=3 k$ and $\operatorname{dim} L=8(k-1)+3=$ $8(n / 3-1)+3=8\left(\left(2^{t-1}+1\right) / 3-1\right)+3=\left(2^{t+2}-7\right) / 3$. If $t$ is odd, then $n=3 k+2$ and $\operatorname{dim} L=8(k-1)+9=8((n-2) / 3-1)+9=8\left(\left(2^{t-1}-1\right) / 3-1\right)+9=\left(2^{t+2}-5\right) / 3$. 


\section{REFERENCES}

[1] W. Burnside. On some properties of groups whose orders are powers of primes. Proc. Lond. Math. Soc., 11:225-243, 1913.

[2] W. Burnside. On some properties of groups whose orders are powers of primes. Proc. Lond. Math. Soc., 13:6-12, 1914

[3] S. Evans-Riley, M. F. Newman, and C. Schneider. On the solvable length of groups of primepower order. Bull. Aus. Math. Soc., 59:343-346, 1999.

[4] S.P. Glasby. Subgroups of the upper triangular matrix group with maximal derived length and minimal number of generators. Groups St. Andrews in Bath, 1997. London Math. Soc. Lecture Notes Ser 260. Cambridge Univ. Press, Cambridge 1999.

[5] P. Hall. A contribution to the theory of groups of prime power order. Proc Lond. Math. Soc., 36:29-95, 1934. 